UDC 524, 531

PACS 04.20.- q, 04.20.Dw

DOI: $10.22363 / 2658-4670-2019-27-3-231-241$

\title{
Geodesic motion near self-gravitating scalar field configurations
}

\author{
Ivan M. Potashov, Julia V. Tchemarina, Alexander N. Tsirulev \\ Faculty of Mathematics \\ Tver State University \\ 35 Sadovy Pereulok, Tver 170002, Russian Federation
}

(received: December 12, 2019; accepted: December 23, 2019)

We study the geodesics motion of neutral test particles in the static spherically symmetric spacetimes of black holes and naked singularities supported by a selfgravitating real scalar field. The scalar field is supposed to model dark matter surrounding some strongly gravitating object such as the centre of our Galaxy. The behaviour of timelike and null geodesics very close to the centre of such a configuration crucially depends on the type of spacetime. It turns out that a scalar field black hole, analogously to a Schwarzschild black hole, has the innermost stable circular orbit and the (unstable) photon sphere, but their radii are always less than the corresponding ones for the Schwarzschild black hole of the same mass; moreover, these radii can be arbitrarily small. In contrast, a scalar field naked singularity has neither the innermost stable circular orbit nor the photon sphere. Instead, such a configuration has a spherical shell of test particles surrounding its origin and remaining in quasistatic equilibrium all the time. We also show that the characteristic properties of null geodesics near the centres of a scalar field naked singularity and a scalar field black hole of the same mass are qualitatively different.

Key words and phrases: geodesic, black hole, naked singularity, scalar field

\section{Introduction}

In recent years new astrophysical observations give us convincing evidence for the presence of strongly gravitating objects in the center of most of normal galaxies. These objects are commonly identified with supermassive black holes, but the modern astrophysical data are not quite enough to exclude other possibilities, such as naked singularities, boson stars, and wormholes. For example, the shadow in the centre of the galaxy M87 and the real image of the photon ring around the shadow, observed by the Event Horizon Telescope collaboration this year, have been immediately interpreted as the existence of the photon sphere and, consequently, the event horizon [1]. However, it is shown in Ref. [2] within a simple model that a naked singularity can also have

(C) Potashov I. M., Tchemarina J. V., Tsirulev A. N., 2019

This work is licensed under a Creative Commons Attribution 4.0 International License http://creativecommons.org/licenses/by/4.0/ 
both the shadow and the photon sphere. In fact, a natural way to distinguish between the different types of the gravitating objects is to explore spacetime geometry through the study (both observationally and theoretically) of the motion of test particles and light rays near the centres of galaxies [3]-[5].

The observational efficiency directly depends on a model in which the astrophysical data for the central objects will be interpreted. First, one should not think of the central objects in galaxies as being in vacuum, because dark matter is mainly concentrated around them. Another problem is that the nature of dark matter and its distribution near galactic centres remain unknown at present. This means that a meaningful interpretation of the observations should be based on an appropriate mathematical model of the central regions: we model dark matter by a nonlinear scalar field which is assumed to be minimally coupled to gravity. Our aim is to compare the behaviour of timelike and null geodesics for a scalar field black hole and a scalar field naked singularity of the same mass. For such configurations, the general properties of timelike geodesics were studied in Refs. [6]-[8]. In this paper, we focus our attention on the comparison of bound trajectories of massive test particles and light rays in the immediate vicinity of the centres of scalar field black holes and scalar field naked singularities.

The paper is organised as follows. In Section 2 we describe the necessary mathematical background for static, spherically symmetric scalar field configurations restricting our attention to the case of the minimal coupling between curvature and a real scalar field. In Section 3 we discuss general features of bound and unbound orbits of free neutral massive and massless particles. In Section 4 we consider a simple example which illustrates some characteristic features of the photon orbits of scalar field black holes and scalar field naked singularities in comparing with the orbits of massive test particles.

In this paper, we use the geometrical system of units with $G=c=1$ and adopt the metric signature $(+---)$. In tensor notation, we use the summation convention over repeated indices, and Greek indices take the values $0,1,2,3$.

\section{Self-gravitating spherically symmetric scalar field configurations}

We begin with the action

$$
\Sigma=\frac{1}{8 \pi} \int\left(-\frac{1}{2} R+\langle d \phi, d \phi\rangle-2 V(\phi)\right) \sqrt{|g|} d^{4} x,
$$

where $\phi$ is a real scalar field, $R$ is the scalar curvature, $V(\phi)$ is a self-interaction potential, and the angle brackets denote the scalar product with respect to the spacetime metric. The metric of a spherically symmetric spacetime in the Schwarzschild-like coordinates can be written in the form

$$
d s^{2}=A d t^{2}-\frac{d r^{2}}{f}-r^{2}\left(d \theta^{2}+\sin ^{2} \theta d \varphi^{2}\right),
$$


where the metric functions $A$ and $f$ depend only on the radial coordinate $r$. For the metric function $A$, it is convenient to make the substitution

$$
A(r)=f(r) \mathrm{e}^{2 F(r)},
$$

so that the Einstein-Klein-Gordon equations take the form

$$
\begin{gathered}
-\frac{f^{\prime}}{r}-\frac{f-1}{r^{2}}={\phi^{\prime}}^{2} f+2 V, \\
\frac{f}{r}\left(2 F^{\prime}+\frac{f^{\prime}}{f}\right)+\frac{f-1}{r^{2}}=\phi^{\prime 2} f-2 V, \\
-f \phi^{\prime \prime}-\frac{\phi^{\prime}}{2} f^{\prime}-\phi^{\prime} f\left(F^{\prime}+\frac{1}{2} \frac{f^{\prime}}{f}+\frac{2}{r}\right)+\frac{d V}{d \phi}=0,
\end{gathered}
$$

where a prime denotes differentiation with respect to $r$.

By adding equations (1) and (2), we obtain

$$
F^{\prime}=r \phi^{\prime 2} \text {. }
$$

Now we can eliminate $F^{\prime}$ from the two other equations and, as a result, solve those in the form of quadratures [9]-[15]. For our goal, we will employ the integral formulae obtained in Ref. [13]:

A general static, spherically symmetric, asymptotically flat solution of equations (1)-(3) with an arbitrary self-interaction potential is given by the quadratures

$$
\begin{gathered}
F(r)=-\int_{r}^{\infty}{\phi^{\prime}}^{2} r d r, \quad \xi(r)=r+\int_{r}^{\infty}\left(1-\mathrm{e}^{F}\right) d r, \\
A(r)=2 r^{2} \int_{r}^{\infty} \frac{\xi-3 M}{r^{4}} \mathrm{e}^{F} d r, \quad f(r)=\mathrm{e}^{-2 F} A, \\
\tilde{V}(r)=\frac{1}{2 r^{2}}\left(1-3 f+r^{2}{\phi^{\prime}}^{2} f+2 \mathrm{e}^{-F} \frac{\xi-3 M}{r}\right),
\end{gathered}
$$

where the parameter $M$ is the Schwarzschild mass.

It is important to stress that each solution of equations (1)-(3) satisfies these quadratures independently of the monotonicity of the field function.

In order to use these quadratures, we will work with a specially defined function $\xi(r)$ which must satisfy the asymptotic condition

$$
\xi=r+O(1 / r), \quad r \rightarrow \infty,
$$

and then will sequentially find the metric functions $\left(\mathrm{e}^{F}=\xi^{\prime}\right)$, the scalar field (by integrating (4)), the function $\tilde{V}(r)$, and the self-interaction potential $V(\phi)=\tilde{V}(r(\phi))$. This method is commonly known as 'the inverse problem method for self-gravitating scalar field configurations'. It is also important to stress that we consider the scalar field as a phenomenological model of dark matter rather than as a really existing fundamental field. Note that 
we could include the cosmological constant in the potential as the additional term $\Lambda / 2$, but its contribution to the geometry of the central region would be negligible. The absence of the cosmological constant simply means that $V(\phi(\infty))=0$. Therefore, the so-called 'no-hair theorem' is not essential in the current astrophysical context.

Using quadratures (5) and (6), we can write algebraically independent components of the curvature in the orthonormal basis, associated with the metric (2), in the form

$$
\begin{gathered}
R_{0101}=\phi^{\prime 2} f-\frac{f-1}{r^{2}}, \quad R_{2323}=\frac{f-1}{r^{2}} \\
R_{0202}=R_{0303}=-\frac{f}{r^{2}}+\mathrm{e}^{-F} \frac{\xi-3 M}{r^{3}} \\
R_{1212}=R_{1313}=\frac{f}{r^{2}}-{\phi^{\prime}}^{2} f-\mathrm{e}^{-F} \frac{\xi-3 M}{r^{3}} .
\end{gathered}
$$

On the other hand, in spherically symmetric spacetimes, the Kretchmann invariant, $K=R_{\alpha \beta \gamma \delta} R^{\alpha \beta \gamma \delta} / 4$, equals the sum of the squared curvature components and, therefore, diverges if at least one of the curvature components do. Thus, in this case $K$ and the scalar curvature $R$ diverge at $r=0$ for all solutions with the exception of some specific ones satisfying the conditions

$$
\xi(r)=3 M+\mathrm{e}^{F(0)} r+O\left(r^{3}\right), \quad f(0)=1+O\left(r^{2}\right), \quad r \rightarrow \infty .
$$

In the generally accepted manner, we call a solution a naked singularity ( $a$ black hole) if $K$ diverges at $r=0$ and $f>0$ for all $r>0$ (respectively, $f=0$ at some radius $r_{h}>0$ and $f>0$ for all $\left.r>r_{h}\right)$.

For a given nonzero scalar field $\phi(r)$, it follows directly from (5) that $\xi^{\prime}=\mathrm{e}^{F}>0$ for all $r>0$ and $\xi(0)>0$, so that the metric function $A$, given by the quadrature (6), passes through zero and becomes negative as $r \rightarrow 0$ if and only if $3 M>\xi(0)$. In other words, the corresponding configuration of mass $M$ will be a naked singularity or a black hole if and only if

$$
0<3 M<\xi(0) \text { (naked singularities) or } 3 M>\xi(0) \text { (black holes) }
$$

respectively. In what follows we deal only with 'generic' configurations and do not consider the special (fine-tuned) case $3 M=\xi(0)$; the latter leads to a naked singularity or a regular solution.

The geometrical system of units $(G=c=1)$ does not fix a unit of length. On the other hand, the geodesic structure of spacetime is scale invariant at the classical level, and the solution $(6)-(7)$ is invariant under the scale transformations

$$
r \rightarrow r / \lambda, \quad M \rightarrow M / \lambda, \quad V \rightarrow \lambda^{2} V, \quad \lambda>0
$$

so that we can use an arbitrary unit of length. By applying $\lambda=M$ in this transformation, we can take, as it is usually done in general relativity, the mass of a scalar field configuration as the current unit of length. Thus, without loss of generality, we suppose everywhere below that $M=1$. 


\section{Geodesic motion around scalar field configurations}

In all stationary spherically symmetric spacetimes we have the conserved energy and angular momentum of a test particle. Together with the constancy condition for the norm of the four-velocity, this implies the existence of three integrals of motion. For the metric (2) they can be written in the form

$$
\begin{gathered}
\frac{d t}{d s}=\frac{E}{A}, \quad \frac{d \varphi}{d s}=\frac{J}{r^{2}}, \quad\left(\frac{d r}{d s}\right)^{2}=\mathrm{e}^{-2 F}\left(E^{2}-V_{\text {eff }}\right), \\
V_{\text {eff }}=A\left(k+\frac{J^{2}}{r^{2}}\right),
\end{gathered}
$$

where $V_{\text {eff }}(r)$ is the effective potential, $E$ and $J$ are, respectively, the specific energy and the specific angular momentum of a test particle, and $k=0$ or $k=1$ depending on whether we are considering null or timelike geodesics; for null geodesics, $s$ is an arbitrary geodesic parameter, but not the interval.

For any asymptotically flat spacetime and for any value of $J, V_{\text {eff }} \rightarrow 0$ or $V_{\text {eff }} \rightarrow 1$ as $r \rightarrow \infty$ depending on whether $k=0$ or $k=1$, respectively. Another difference between null and timelike geodesics which is more interesting is that the behaviour of a null geodesic effectively depends only on the impact parameter $b$, so that

$$
\left(\frac{d r}{d s}\right)^{2}=E^{2} \mathrm{e}^{-2 F}\left(1-b^{2} \frac{A^{2}}{r^{2}}\right), \quad b=\frac{J}{E}
$$

where the coefficient $E^{2}$ on the right hand side can be eliminated by redefining the parameter: $\lambda=s E$.

It is shown in Ref. [8] that for a scalar field black hole spacetime, defined by the quadratures (5)-(7) and the conditions (8) and $3 M>\xi(0)$, the lapse function $A(r)$ is a strictly increasing function outside the event horizon. In contrast, for a naked singularity spacetime, satisfying the conditions (8) and $3 M<\xi(0), A(r)$ necessarily has at least one minimum in the region $0<r<\infty$. These properties give us a key distinguishing feature for the two types of scalar field configurations: they cause different behaviours of bound orbits close to the centre. The various numerical simulations with scalar field black hole solutions allow us to conclude that the radius of the corresponding innermost stable circular orbit, which is an important observational characteristic for black holes, is of order $3 r_{h}$ (analogously to the vacuum case). On the contrary, a scalar field naked singularity has no innermost stable circular orbit but has a unique degenerated static orbit, which has $J=0$ and is located at $r=r_{0}$, where $r_{0}$ is the unique solution of the equation $A^{\prime}(r)=0$. From the point of view of a distant observer resting relative to the centre, a test particle remains at rest in the static orbit all time. Particles in such a static orbit, together with particles having zero or small specific angular momentum and specific energy $E^{2} \gtrsim A\left(r_{0}\right)$, can make up a spherical shell consisting of cold gas or fluid. For a distant observer, this shell would look like a shadow similar to that of a black hole. 
The expressions for trajectories of timelike and null geodesics can be expressed directly from (12) and (14). They are, respectively,

$$
\varphi=J \int \frac{\mathrm{e}^{F}}{r^{2} \sqrt{E^{2}-V_{\text {eff }}}} d r \text { and } \varphi=b \int \frac{\mathrm{e}^{F}}{r^{2} \sqrt{1-b^{2} A^{2} / r^{2}}} d r .
$$

\section{Geodesics: analytic examples}

For the sake of brevity and simplicity, we will explore a fully analytic, one-parameter family of solutions defined by the functions

$$
\xi=\sqrt{r^{2}+2 a r+5 a^{2}}-a, \quad \mathrm{e}^{F}=\xi^{\prime}=\frac{r+a}{\sqrt{r^{2}+2 a r+5 a^{2}}},
$$

which uniquely determine the metric function $A(r)$ and the scalar field $\phi(r)$. By direct integration in (6), we obtain

$$
A=1+\frac{2 a}{3 r}-2 \frac{a+3 m}{15 a}\left\{\frac{\sqrt{r^{2}+2 a r+5 a^{2}}}{r}\left(1+\frac{r}{a}-\frac{r^{2}}{a^{2}}\right)+\frac{r^{2}}{a^{2}}\right\},
$$

where $a$ is the parameter of 'intensity' of the scalar field. Using (9)-(11) we find that the Kretchmann invariant diverges at the centre. In accordance with the condition $3 M<\xi(0)$, the inequality $a>3 /(\sqrt{5}-1)$ determines the subfamily of scalar field naked singularities.

The results of numerical simulation of geodesics are presented in Figures 1-3.
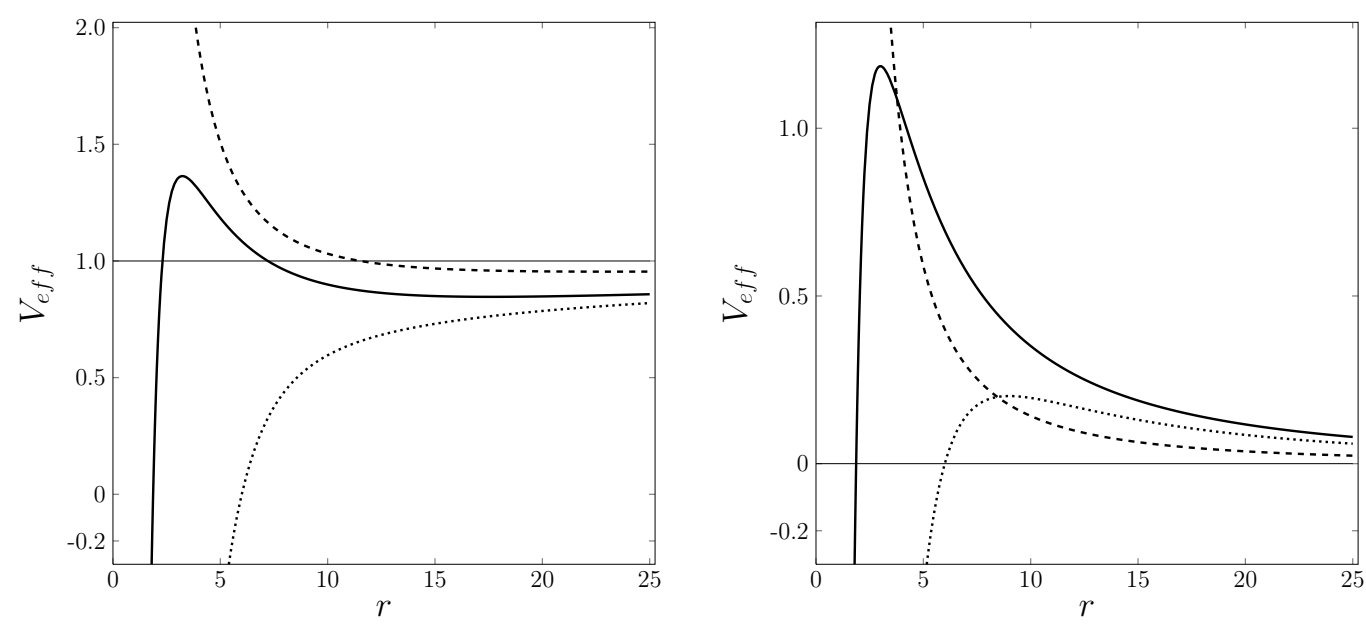

Figure 1. The left (right) panel shows the effective potentials of massive particles (of photons): for the scalar field black hole (solid curve) with $a=6, M=3, J=7.2$ and naked singularity (dashed curve) with $a=6, M=1, J=7.2$, and for the Schwarzschild black hole (dotted curve) with $M=3, J=7.2$ 

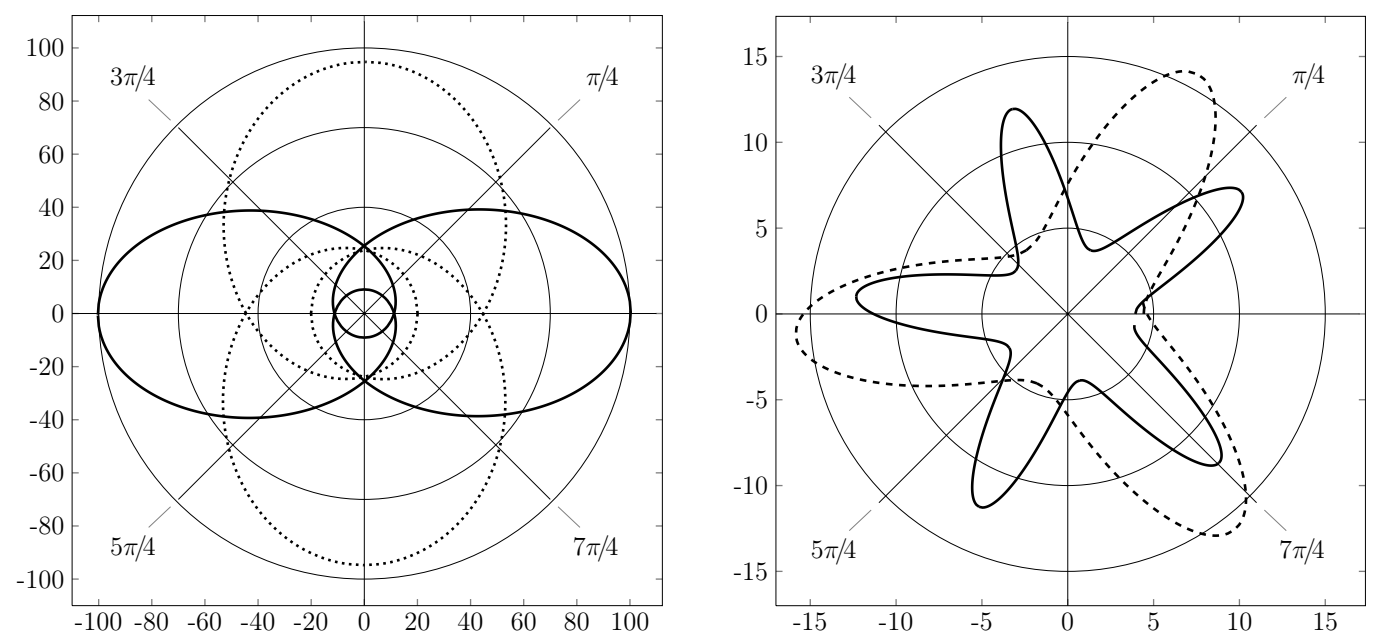

Figure 2. The shape of orbits of massive particles. Left panel: the scalar field black hole with $a=5, M=3, J=9, E^{2}=0.948$ (solid curve) and the Schwarzschild black hole (dotted curve) with $M=3, J=12, E^{2}=0.9517$. Right panel: the scalar field naked singularities with $a=5, M=1$ and $J=0.5,, E^{2}=0.88$ (solid curve) and $J=1, E^{2}=0.9011$ (dashed curve)
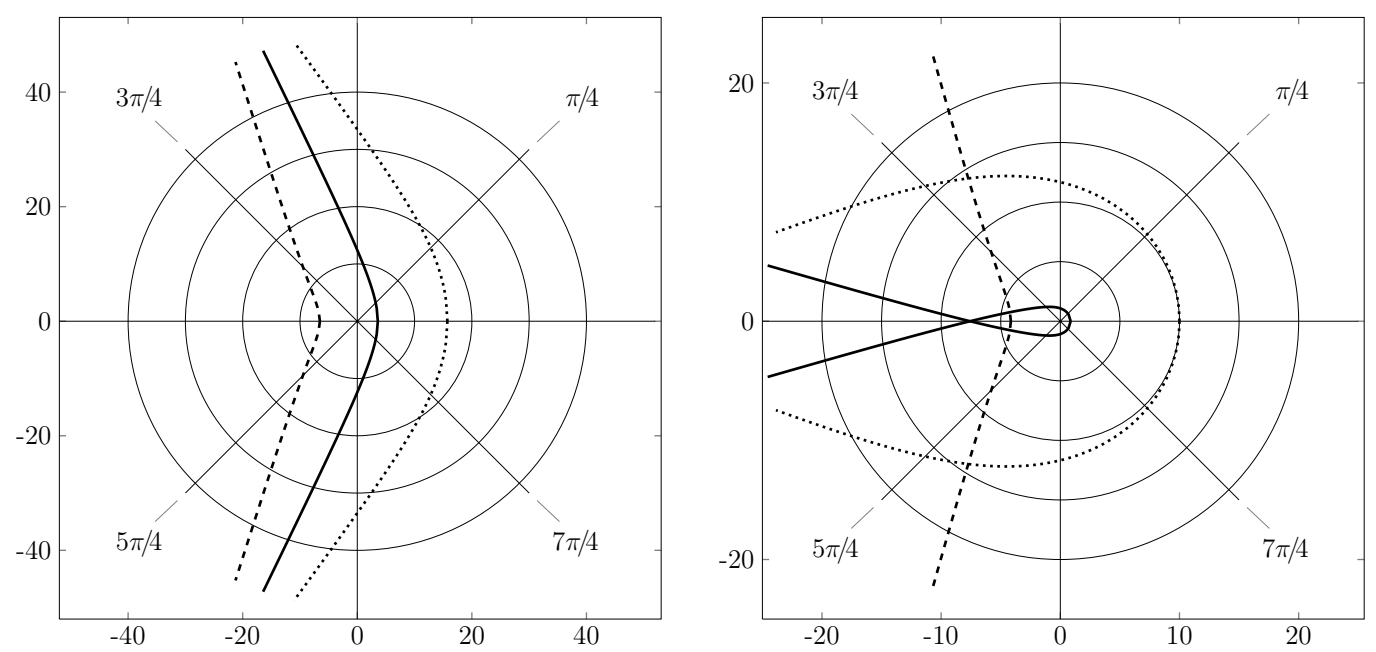

Figure 3. The shape of orbits of photons. Left panel: the scalar field black hole with

$a=7, M=3, b=6$ (solid curve), the scalar field naked singularity with $a=7, M=1, b=6$ (dashed curve), and the Schwarzschild black hole (dotted curve) with $M=3, b=20$. Right panel: analogously to the left panel, but with parameters $a=7, M=3, b=2.5$ (solid curve), $a=7, M=1, b=2$ (dashed curve), and $M=3, b=12$ (dotted curve)

The typical effective potentials are shown in Figure 1, where the difference between timelike and null geodesics is obvious: in particular, the effective potentials of photons in the spacetime of a scalar field naked singularity do 
not have extrema, so that there does not exist the photon sphere. In Figure 2, the shape of geodesics for massive test particles are plotted. The shape of an orbit depends on the specific angular momentum $J$ and the specific energy $E$ of a test particle. Numerical simulation shows that the number of oscillations per revolution decreases with increasing $J$ when the value of $E$ is fixed, as well as with decreasing $E$ when the value of $J$ is fixed. Figure 3 presents photon orbits. The difference in the behaviour of null geodesics is obvious.

\section{Conclusions}

The observations of timelike and null geodesics very close to the centres of galaxies are of great importance, allowing us to study the spacetime geometry near the centres as well as to understand the nature of the central supermassive self-gravitating objects. The available astrophysical data are so far insufficient to identify the strongly gravitating objects and even to definitely distinguish between black holes and naked singularities. However, we can hope that the future development of the spatial resolving power of precise astronomical instruments will be sufficient to observe the central region of Sgr $\mathrm{A}^{*}$ (about $15 M \approx 0.15$ mas). In particular, the facilities of the Event Horizon Telescope are expected to achieve the required sensitivity within the next fifteen years [16], [17].

In this paper, we study (in a fully analytical manner) the characteristic features of the trajectories of null and timelike geodesics in the neighbourhoods of static, spherically symmetric scalar field black holes and naked singularities, having in mind the centre of a galaxy surrounded by dark matter. It turns out that a scalar field black hole, analogously to a Schwarzschild black hole, has the innermost stable circular orbit and the (unstable) photon sphere, but their radii are always less than the corresponding ones for the Schwarzschild black hole of the same mass; moreover, these radii can be arbitrarily small. We show that a scalar field naked singularity has a positive Schwarzschild mass (as opposed to vacuum naked singularities), but has neither the innermost stable circular orbit nor the photon sphere. Instead, such a configuration has a spherical shell of test particles surrounding its origin and remaining in quasistatic equilibrium all the time. We have shown that the characteristic properties of null geodesics near the centres of a scalar field naked singularity and a scalar field black hole of the same mass are qualitatively different. In particular, the quasistatic equilibrium shell can consists of sufficiently cold particles and be observed as a dark shadow surrounded by a photon image. It means that the observation of only the central shadow, circled by a bright ring, is not by itself enough to identify with confidence the central objects. In fact, a key role in the identification of these objects plays observations of the orbits of stars and gas clouds very close to the centre. Note also (this is important for astrophysical applications) that the behaviour of geodesics in the region $r \gtrsim 15 m$, where the metrics of a scalar field configuration becomes close to the Schwarzschild one, is qualitatively similar to each other and quantitatively almost the same, so that the observations of the geodesic motion in this region are not sufficiently informative. 


\section{References}

[1] The EHT collaboration, "First M87 Event Horizon Telescope Results. I. The Shadow of the Supermassive Black Hole," The Astrophysical Journal Letters, vol. 875, no. 1, 2019. DOI: 10.3847/2041-8213/ab0ec7.

[2] R. Shaikh, P. Kocherlakota, R. Narayan, and P. S. Joshi, "Shadows of spherically symmetric black holes and naked singularities," Monthly Notices of the Royal Astronomical Society, vol. 482, no. 1, pp. 52-64, 2018. DOI: $10.1093 / \mathrm{mnras} / \mathrm{sty} 2624$.

[3] V. I. Dokuchaev and Y. N. Eroshenko, "Weighing of the dark matter at the center of the Galaxy," JETP Letters, vol. 101, no. 12, pp. 777-782, 2015. DOI: $10.1134 / \mathrm{S} 0021364015120048$.

[4] A. Hees et al., "Testing General Relativity with stellar orbits around the supermassive black hole in our Galactic center," Physycal Review Letters, vol. 118, no. 22, p. 211101, 2017. DOI: 10.1103/PhysRevLett. 118.211101.

[5] A. V. Zakharov, "Constraints on tidal charge of the supermassive black hole at the Galactic Center with trajectories of bright stars," European Physical Journal C, vol. 78, p. 689, 2018. DOI: 10.1140/epjc/s10052018-6166-5.

[6] M. De Laurentis, Z. Younsi, O. Porth, Y. Mizuno, and L. Rezzolla, "Test-particle dynamics in general spherically symmetric black hole spacetimes," Physical Review D, vol. 97, no. 10, p. 104 024, 2018. DOI: 10.1103/PhysRevD.97.104024.

[7] G. Z. Babar, A. Z. Babar, and Y. K. Lim, "Periodic orbits around a spherically symmetric naked singularity," Physical Review D, vol. 96, no. 8, p. 084 052, 2017. DOI: 10.1103/PhysRevD.96.084052.

[8] I. M. Potashov, J. V. Tchemarina, and A. N. Tsirulev, "Bound orbits near scalar field naked singularities," European Physical Journal C, vol. 79, p. 709, 2019. DOI: 10.1140/epjc/s10052-019-7192-7.

[9] K. A. Bronnikov and G. N. Shikin, "Spherically symmetric scalar vacuum: no-go theorems, black holes and solitons," Gravitation and Cosmology, vol. 8, pp. 107-116, 2002.

[10] V. V. Nikonov, J. V. Tchemarina, and A. N. Tsirulev, "A two-parameter family of exact asymptotically flat solutions to the Einstein-scalar field equations," Classical and Quantum Gravity, vol. 25, no. 13, p. 138001 , 2008. DOI: $10.1088 / 0264-9381 / 25 / 13 / 138001$.

[11] J. V. Tchemarina and A. N. Tsirulev, "Spherically symmetric gravitating scalar fields. The inverse problem and exact solutions," Gravitation and Cosmology, vol. 15, pp. 94-95, 2009.

[12] M. Azreg-Ainou, "Selection criteria for two-parameter solutions to scalar-tensor gravity," General Relativity and Gravitation, vol. 42, no. 6, pp. 1427-1456, 2010. DOI: 10.1007/s10714-009-0915-6.

[13] D. A. Solovyev and A. N. Tsirulev, "General properties and exact models of static selfgravitating scalar field configurations," Classical and Quantum Gravity, vol. 29, no. 5, p. 055 013, 2012. DOI: 10.1088/02649381/29/5/055013. 
[14] P. V. Kratovitch, I. M. Potashov, J. V. Tchemarina, and A. N. Tsirulev, "Topological geons with self-gravitating phantom scalar field," Journal of Physics: Conference Series, vol. 934, no. 1, p. 012 047, Dec. 2017. DoI: 10.1088/1742-6596/934/1/012047.

[15] I. M. Potashov, J. V. Tchemarina, and A. N. Tsirulev, "Bound orbits near black holes with scalar hair," Journal of Physics: Conference Series, vol. 1390, no. 1, p. 012 097, Nov. 2019. DOI: 10.1088/1742-6596/1390/ $1 / 012097$.

[16] S. Gillessen et al., "An update on monitoring stellar orbits in the galactic center," The Astrophysical Journal, vol. 837, no. 1, p. 30, 2017. DOI: $10.3847 / 1538-4357 /$ aa5c41.

[17] C. Goddi et al., "BlackHoleCam: fundamental physics of the Galactic center," International Journal of Modern Physics D, vol. 26, no. 2, p. 1730 001, 2017. DOI: 10.1142/S0218271817300014.

\section{For citation:}

I. M. Potashov, J. V. Tchemarina, A. N. Tsirulev, Geodesic motion near selfgravitating scalar field configurations, Discrete and Continuous Models and Applied Computational Science 27 (3) (2019) 231-241. DOI: 10.22363/26584670-2019-27-3-231-241.

\section{Information about the authors:}

Ivan M. Potashov - Master of Science in Mathematics, Assistant of Department of General Mathematics and Mathematical Physics (e-mail: potashov . imetversu.ru, ORCID: https://orcid.org/0000-0002-7597-4366, Scopus Author ID: 57200106410)

Julia V. Tchemarina - Candidate of Physical and Mathematical Sciences, Assistant of professor of Department of General Mathematics and Mathematical Physics (e-mail: chemarina. yv@tversu.ru, ORCID: https://orcid.org/0000-0002-9002-887X, Scopus Author ID: 24460923700)

Alexander N. Tsirulev - Doctor of Physical and Mathematical Sciences, Professor of Department of General Mathematics and Mathematical Physics (e-mail: tsirulev. an@tversu.ru, ORCID: https://orcid.org/0000-0003-4168-3613, Scopus Author ID: 16409936300) 
УДК 524, 531

PACS 04.20.-q, 04.20.Dw

DOI: $10.22363 / 2658-4670-2019-27-3-231-241$

\title{
Геодезическое движение вблизи самогравитирующих конфигураций скалярного поля
}

\author{
И. М. Поташов, Ю. В. Чемарина, А. Н. Цирулев \\ Математический факультет \\ Тверской государственный университет \\ Садовый пер., д. 35, г. Тверь, 170002, Россия
}

В работе изучается геодезическое движение нейтральных пробных частиц в пространстве-времени статических сферически-симметричных чёрных дыр и голых сингулярностей, порождённых самогравитирующим скалярным полем. Предполагается, что скалярное поле моделирует тёмную материю, окружающую некоторый объект с сильным гравитационным полем, такой как центр нашей Галактики. Поведение времениподобных и изотропных геодезических, проходящих очень близко к центру такой конфигурации, в решающей степени зависит от типа пространства-времени. Оказывается, что скалярно-полевая чёрная дыра, подобно чёрной дыре Шварцшильда, имеет последнюю устойчивую круговую орбиту и (неустойчивую) фотонную сферу, но их радиусы всегда меньше соответствующих радиусов для чёрной дыры Шварцшильда той же массы; кроме того, эти радиусы могут быть сколь угодно малыми. Напротив, голая сингулярность, порождённая скалярным полем, не имеет ни последней устойчивой круговой орбиты, ни фотонной сферы. Вместо этого такая конфигурация имеет сферическую оболочку из частиц, окружающую её центр и всё время находящуюся в квазистатическом равновесии. Также показано, что характерные свойства изотропных геодезических вблизи центра скалярного поля голой сингулярности и центра скалярного поля чёрной дыры, имеющих одинаковую массу качественно различны.

Ключевые слова: геодезическая, чёрная дыра, голая сингулярность, скалярное поле 